\title{
Understanding Black and Minority Ethnic service user's experience of racism as part of the assessment, formulation and treatment of mental health problems in cognitive behaviour therapy
}

\author{
Andrew Beck \\ East Lancs Child and Adolescent Service, University of Manchester
}

Received 21 November 2017; Accepted 11 September 2018

\begin{abstract}
Experiences of racism can be a cumulative risk factor for developing mental health problems. Cognitive Behaviour Therapists working with Black and Minority Ethnic (BME) service users should be confident in their ability to establish the necessary rapport to ask about these experiences and be able to incorporate this information into longitudinal formulations and as part of maintenance cycles. This paper sets out guidelines as to how to do this as part of a wider engagement process.
\end{abstract}

Key words: BAME, case formulation, CBT, racism

\section{Background}

Recent research from the University of Manchester has shown that the more someone from a Black and Minority Ethnic (BME) background experiences racism, the more likely they are to experience mental health problems (Wallace et al., 2016). This study suggests that racist incidents have a cumulative effect over the course of someone's lifetime and that the more someone experiences these, the more likely they are to develop a mental health problem. It is also likely that particular incidents could have a precipitating effect on mental health problems, meaning that the onset of a particular presenting problem may be linked to a specific incident of racist abuse or discrimination.

There is also good evidence that people from BME communities in the UK are experiencing more racial harassment since the beginning of the Brexit campaign, with some monitoring organizations reporting a fivefold increase of incidents. There has also been a growing concern about increased anti-Semitism and Islamophobia that preceded the Brexit campaign.

What this means for Cognitive Behaviour Therapists is that there is a good chance that BME service users will have experienced racism over their lifetime and that this might be one of several causal factors linked to the development of their mental health problems or linked to a recent deterioration in their well-being.

These experiences of racism can take many forms, including overt violent attacks, verbal abuse or micro-aggressions. These can take several forms and can include exposure to negative 
views about a particular ethnic group not necessarily made directly to or about the person, name-calling, being avoided in social encounters or excluded from them, rudeness, subtle snubs and insults that fall below the radar of explicit racism but which convey inferiority or marginalizing the experiences. There is no current widely accepted definition of microaggression and research into this area remains scant, suggesting that it is a concept that should be considered but held lightly by researchers and therapists until we have a clearer picture of what this means and how it can cumulatively impact on people (Lilienfeld, 2017).

Service users are also likely to have experienced racism that has impacted on their life chances and access to jobs, housing, education and other opportunities, and may be aware of and troubled by this to different extents. Service users from a wide variety of cultures and faiths experience these forms of direct and indirect discrimination including people of South Asian, non-British White European particularly those from Eastern Europe, Jewish and Black African, Black Caribbean and Black British backgrounds.

This paper is written to provide Cognitive Behaviour Therapists from a White British cultural background with a framework to begin to confidently work with BME service users to think about the impact of racism on their mental health.

In order to begin to ask service users about their experiences of racism, therapists need to start by asking them about their ethnic backgrounds.

\section{Asking about culture, ethnicity and faith}

We know from past research (Messent and Murrell, 2003) that many White therapists find it difficult to ask service users about their ethnic background and to begin to think about culture and context either as part of getting to know the lives of the people we are working with or as information that might help in gaining an understanding of the presenting problem. One of the most common reasons that therapists give for this is being worried about getting this discussion wrong and giving the service user the impression that they are racist or culturally uninformed. Therapists tend to avoid topics that are anxiety-provoking so it is understandable that we sometimes avoid conversations in therapy where we are worried about the outcome (Scherr et al., 2015).

It is also possible that therapists using manualized approaches may not think to ask about the wider context of racism and discrimination. This might be the case in Cognitive Behavioural Therapy (CBT) where the disorder-specific models make little or no reference to the social contexts of service users and their experiences of discrimination as predisposing, precipitating or maintaining factors in formulations. It is equally likely to be an issue in psycho-dynamically informed therapy where there has historically been very little consideration of cross-cultural difference or the possibility of chronic experiences of racism impacting on someone's psychological development. Although systemic therapies have historically had more developed and reflective approaches to thinking about culture and diversity than CBT, there has still not been a great deal of emphasis on the need to directly ask service users about their experiences of racism.

What service users say very clearly is that not only do they want to be asked about their ethnic background but that when therapists do so it can strengthen the therapeutic relationship and go a long way towards giving them confidence that a White therapist can understand the social contexts of the problems they are bringing to therapy (Gurpinar-Morgan et al., 2014). 
This positive contribution to developing the therapeutic relationship can also provide the basis for more detailed discussions about culture, context and experiences of racism.

With this in mind it seems useful to summarize some key points from what little research has been written on this topic so that Cognitive Behavioural Therapists can feel confident about raising this issue with service users and incorporating this information into formulations and treatment.

Firstly therapists need to ask about the culture and background of service users. This is not something that should be done before some degree of trust and the beginnings of a therapeutic relationship has been established. Cognitive behavioural therapists will of course use the core therapy skills of warmth and positive regard to establish the basis for a working relationship, although it is likely that even these core skills need a degree of adaptation when working across cultures (Rathod et al., 2015; pp. 99-105).

Once some rapport and trust has been established, the therapist can begin to look for opportunities to ask about culture, ethnicity and context. Service users are fairly consistent in saying that acknowledging the difference between the ethnicity of the therapist and service user helps this discussion and it can be a useful way for therapists to introduce the topic of ethnicity and communicate that they recognize and value diversity. This acknowledgement could be as simple as saying something like "As I am from a different cultural background to you it would really help me to understand you and your difficulties better if I could ask about your background.' Sometimes it can be helpful to allow more room for service users to think about a request like this and to say 'Would it be okay if I asked you a question about your background?' It may be easier for service users who do not want to have this discussion to say so in response to a less direct question like this although in my experience people have always been happy to explain cultural and contextual issues to me as a White therapist once this initial rapport has developed, differences acknowledged and permission granted.

Service users will often give the therapist cues and openings for these discussions (Gurpinar-Morgan et al., 2014). This might include mentions of their wider family, how they have spent the time since their last appointment (for example mentioning that they spent one day of the weekend at a family function or religious event) or communicating something as simple as having eaten a particular food that can be seen as a signifier of their ethnicity. Cognitive Behavioural Therapists can respond to these cues by saying something like 'Could you tell me a bit more about your family background?' This provides the service user with an opportunity to say as much or as little as they are comfortable with saying at that moment in time. In research by Gurpinar-Morgan et al. (2014), service users were clear that when they did not provide these cues it was still important for therapists to ask about culture and context. It might be that service users do not provide these openings because they do not expect therapists to be interested in this topic or because they are worried about how a discussion regarding this topic might progress.

Another very helpful way into these sorts of discussions is for the therapist to say something like 'It would be really helpful for me to know a little bit more about who is at home. Could we sketch out a family tree?' Developing a genogram with service users then provides a good opportunity for further discussions about family life, culture and context which can add considerably to a CBT formulation (as considered in detail in Beck, 2016). Co-developing a genogram gives an opportunity to ask about a wide range of factors to do with someone's background including the migration histories of family members, the degree that different 
family members are acculturated, what languages are spoken at home and the importance of different family members in the life of the service user.

Discussions about ethnicity and culture can take place over several appointments and as a bond of trust is developed and strengthened more in-depth topics can be considered. If this topic is initially anxiety provoking for the therapist or service user it is likely to be the case that the feared consequence of the discussion does not happen and that considering these issues improves the therapeutic relationship to such an extent that further exploration becomes possible. A good rule of thumb for therapists is to only ask for as much detail as they need to in order to understand the life of the service user enough to help them overcome the problems they have come to therapy with. It can be tempting to use therapy time to learn about a new culture and become educated about a world that might seem otherwise inaccessible, but some care should be taken about how much is explored. It is also possible that there will be views, values and practices discussed that therapists might find difficult and that these might elicit strong emotional reactions or unhelpful beliefs about a cultural group. A good therapist will recognize these responses and use supervision as a place to think about them, although this assumes access to a supervisor who is seen as competent in thinking this through with the therapist.

It is also worth remembering that many service users from Muslim faith backgrounds will be very aware of programmes within mental health services to identify service users at risk of radicalization and to refer them to counter-terrorism programmes. Bhui (2016) has written about how an awareness of this agenda in health services might make Muslim service users wary of the motives of mental health professionals in asking about their faith and religious observance, at least until therapists have communicated an understanding of and respect for their faith and background.

\section{Asking about racism once trust has developed}

Once discussions about background and ethnicity have taken place it is likely that enough trust and positive regard will have been built up for therapists to begin to ask questions about a BME service user's experiences of racism. This could potentially be an uncomfortable experience for both the therapist and the service user. For service users discussing racism might trigger feelings of shame or initially exacerbate symptoms of depression or anxiety. When the racist incident was violent in nature it might have been the trigger for developing Post-Traumatic Stress Disorder. Where this is the case service users might have spent a considerable amount of time avoiding thinking about the incident. The service user might also be worried about the degree to which the therapist will be able to hear about their experiences and may have had past experiences of White friends and colleagues not understanding or wanting to hear about their experiences of racism. To some degree the skilful way in which ethnicity, faith and culture have already been discussed should have communicated that the therapist is able to think about this topic; however, it might not necessarily be the case. It is likely that BME therapists will be in a far better position to ask and be told about experiences of racism but not every BME service user will have been in a position to be offered the choice of a BME therapist, so it is important that White therapists feel confident enough to have this conversation.

Therapists may well be anxious about asking this question and feel shame or guilt when a service user describes experiences of racism. This does not mean that the therapist is not a good one, this is a very human response to hearing about discrimination. How a therapist responds to these feelings in session is very much a matter of personal preference. Saying 
something like 'Just hearing about what happened makes me feel awful for you and what you have experienced' can communicate something important about your reactions to hearing about difficult situations. Acknowledging that as a White therapist it is difficult to hear about the way someone from your own community has acted can also be helpful for some service users. Other therapists might find it works better for them and the service user to remain engaged and encouraging but not say anything directly about how that makes them feel. However therapists choose to respond, supervision can be a very useful place to reflect on what this process was like and think about how we might want to respond next time. Patel (2004) has written some extremely helpful guidance for supervisors when considering issues of ethnicity and power in supervision, and Beck (2016) contains a chapter on how this might specifically be applied to supervision in CBT.

There is no right time or definitive way to ask service users about experiences of racism. Service users might give cues or speak about these kinds of experiences without prompting from the therapist, particularly if discussions about culture and ethnicity have given the message that you are a therapist who is comfortable and skilled around discussion of these topics. A direct question can be very helpful when no cues have been given. This might be done as simply as saying something like 'I am wondering what it was like to be from one of the few Black families in your area?' or when someone is describing a difficult situation such as being bullied at school or marginalized at work a question like 'Do you think being from a minority group had any part to play in what happened?'.

One question that may be very helpful is to ask if it is alright to ask about experiences of racism. The use of this question to ask about culture and background in general has been discussed above. This question can be phrased as 'I am wondering if it is okay for me to ask about whether you experienced racism in that situation?'. This gives service users an opportunity to say yes or no to further questioning. For example, after the European Union Brexit referendum BME service users mentioned the outcome of this vote in therapy and this could provide a cue to say 'I wonder if you have noticed being treated differently since the campaign started?'. This could lead to some service users discussing incidents of abuse. For some this may have been the first time in years that this has happened to them. People may report a changed sense of belonging and safety as a result of these or incidents like this happening to people they are close to.

\section{Making use of this information in treatment}

Once the discussion has begun there are many ways that this information can be used in therapy. A collaboratively developed formulation provides an opportunity for therapists to ask service users about how this information can be used to gain a better understanding of presenting problems or where it fits with their current understanding of difficulties. Experiences of racism may be a predisposing or precipitating factor for mental health problems. Bringing this information into the formulation can give a sense of the service user and therapist jointly understanding the wider societal context for someone developing mental health problems. This information can sometimes also be used to understand maintaining factors. For example, a BME service user with social anxiety who is experiencing ongoing racism may talk about thoughts being negatively judged by others on the basis of their ethnic background. Therapists and service users then have the challenge of thinking about the lived reality of the service user where the context for them includes recent racist experiences and 
the degree to which they have realistically appraised how much risk of racist abuse, attacks or negative judgement there is in their environment. This might lead to a discussion about the possibility that part of the presenting problem is that the service user has over-generalized from the fact that some White people are racist and openly hostile towards them, to thinking that most or all White people are racist and believing themselves to be highly vulnerable as a result.

Care should be taken when exploring these ideas not to minimize or discount experiences of or concerns about racism in any way. Hearing about racism in such an intimate way is uncomfortable and therapists might be tempted to downplay experiences so as not to challenge their own beliefs that society is essentially fair and reasonable. Listening to these stories and helping service users understand their problems in the context of these can be an empowering experience for them and it might be that part of their recovery process involves politicizing their experiences and working towards challenging racism at a wider level in society.

\section{Main points}

(1) Experiences of racism have a cumulative impact on the risk of developing mental health problems.

(2) Therapists working with BME service users can use discussions about ethnicity and background to communicate cultural competence to service users.

(3) Once trust has been established, therapists can also ask about experiences of racism.

(4) Understanding these experiences can enhance the therapeutic alliance and inform formulations and treatment.

\section{Acknowledgements}

An abbreviated version of this paper appeared in the September 2016 edition of CBT Today.

\section{Conflicts of interest}

None

\section{Financial support}

None

\section{Ethical approval}

$\mathrm{n} / \mathrm{a}$

\section{References}

Beck A (2016). Transcultural Cognitive Behaviour Therapy for Anxiety and Depression: A Practical Guide. Routledge.

Bhui K (2016). Flash, the emperor and policies without evidence: counter-terrorism measures destined for failure and societally divisive. BJPsych Bulletin 40, 82-84. 
Gurpinar-Morgan A, Murray C, Beck A (2014). Ethnicity and the therapeutic relationship: views of young people accessing cognitive behavioural therapy. Mental Health, Religion and Culture 17, $714-725$.

Lilienfeld SO (2017). Microaggressions: strong claims, inadequate evidence. Perspectives on Psychological Science 12, 138-169.

Messent P, Murrell M (2003). Research leading to action: a study of accessibility of a CAMH service to ethnic minority families. Child and Adolescent Mental Health 8, 118-124.

Patel N (2004). Difference and power in supervision: the case of culture and racism. In I Fleming and L Stern (eds), Supervision and Clinical Psychology: Theory, Practice and Perspectives. BrunnerRoutledge: London.

Rathod S, Kingdon D, Pinninti N, Turkington D, Phiri P (2015) Cultural Adaptation of CBT for Serious Mental Illness: A Guide for Training and Practice. John Wiley and Sons.

Scherr SR, Herbert JD, Forman EM (2015). The role of therapist experiential avoidance in predicting therapist preference for exposure treatment for OCD. Journal of Contextual Behavioral Science 4, $21-29$.

Wallace S, Nazroo J, Bécares L (2016). Cumulative effect of racial discrimination on the mental health of ethnic minorities in the United Kingdom. American Journal of Public Health 106, 1294-1300. 